\title{
Modelling the monotonic and cyclic behaviour of sands using Artificial Neural Networks
}

\author{
Weixian Chen ${ }^{l}$, Andrés Alfonso Peña Olarte ${ }^{l *}$, Roberto Cudmani ${ }^{l}$ \\ ${ }^{1}$ Zentrum Geotechnik, Technical University of Munich, Franz-Langinger-Str. 10, 81245 Munich, Germany
}

\begin{abstract}
In this study artificial neural networks (ANN) are used to simulate the monotonic and cyclic behaviour of sands observed in laboratory tests on Karlsruhe sand under drained and undrained conditions. A genetic algorithm (GA) is used to obtain an optimal framework for the ANN. The results show that the proposed genetic adaptive neural network (GANN) can effectively simulate drained and undrained monotonic triaxial behaviour of saturated sand under isotropic or anisotropic consolidation. The GANN is also able to predict satisfactorily the cyclic behaviour of sands under undrained triaxial test with strain and stress cycles. In addition, GANN is able to distinguish between monotonic drained and undrained conditions by delivering a good prediction when trained with the combined database.
\end{abstract}

\section{Introduction}

Granular soils exhibit under monotonic and cyclic shearing a complex macro-mechanical response. Constitutive models have been developed to describe this complex non-linear stress-strain response, including the failure condition. Constitutive models' laws are of upmost importance for analysing different problems in geotechnical engineering, such as stability analysis of slopes and dams, excavation pits, underground construction, seismic response of soils, etc, in which nonlinearity is taken into account.

In the last decades, many constitutive models for soils have been proposed [1]. The validation of these constitutive models is manifold and can be done in principle through comparison with experimental databases. However, these experimental investigations are time-consuming and expensive. In addition, most of these models involve parameters that either lack of physical meaning or might be very difficult to calibrate with experimental data [2]. Thus, methods that use automatic and machine learning techniques (such as artificial neural networks) to establish the stress-strain relationships from available experimental databases offer a promising alternative [3].

The research of artificial neural networks (ANN) began in the 1940s, but applications in civil engineering did not begin until the late 1980s. Almost at the same time, the wide application spectrum of genetic algorithms (GA) to engineering problems was realized [4]. In 1993, GA was used for the first time in geotechnical engineering to identify discontinuity frequency extrema in fractured rock masses [5]. Since then, GA has been widely used in geotechnical engineering, e.g. the application in parameter identification and stress analysis such as the prediction of soil permeability coefficient [6], estimation of the pressuremeter modulus and limit pressure [7], and the calibration of constitutive models [8], among others.

In this contribution, we apply ANN to simulate both the monotonic and cyclic behaviour of Karlsruhe sand observed in the lab under undrained and drained triaxial conditions [9]. Hereby, the Genetic Adaptive Neural Network (GANN) is used to optimize the ANN to realistically predict the monotonic and cyclic behaviour observed in the experiments with Karlsruhe sand.

\section{Method}

\subsection{Artificial neural networks (ANN)}

ANN is a mathematical model or calculation model that imitates the structure and function of a biological brain neural network, and it is used to estimate or approximate complex mathematical functions. Usually, an artificial neural network is composed of an input layer, an output layer and one or more hidden layers [10].

\subsection{Genetic adaptive neural networks (GANN)}

The back propagation (BP) neural network is based on gradient descent. Although it has accurate optimization capabilities, it has poor search capabilities in the global range, slow convergence, easy to fall into local minimums, and the network structure is not easy to determine, so if this method is used alone, the result may not be optimum [11].

Therefore, this work combines a BP neural network with a GA to obtain a hybrid algorithm that can seek a more accurate solution in the global space to meet the desired target. This allows for finding optimal parameters for

\footnotetext{
* Corresponding author: andres.pena@tum.de

A video is available at https://doi.org/10.48448/ded3-q842
} 
the ANN and improving its learning efficiency. The mean squared error (MSE) between experimental and calculated values is used to evaluate the fitness function and evaluate the fitness of the chromosomes.

For all experimental databases, axial strain, water pressure, initial confining pressure and relative density are chosen as inputs for the GANN. Under undrained test conditions, the outputs are deviatoric stress and mean pressure. For the drained tests, the deviatoric stress, the mean pressure, and the volumetric strain are selected as the outputs. This applies for both training and validation phases.

This study uses the numerical simulation software MATLAB as a working platform to generate the GANN modules. The training function is based on the Levenberg-Marquardt algorithm, and the selection process starts with factor population size $p_{s}=500$, generation $g=5$, and crossover rate $r_{c}=0.6$, and mutation rate $r_{m}=0.1$. The full process is depicted in Fig. 1 .

According to the number of the original sample set $N$, a randomly sorted sequence from 1 to $N$ is generated, and $M$ samples for training are sequentially selected in this sequence. Finally, the GANN is trained with the selected samples. This process is called samples training. In order to show the improvement of ANN's prediction ability with the increase of number of samples used for training, $M$ has been varied from 1 to $N-1$, meaning that samples training has been performed $N-1$ times. This process is called a full training. In order to explore relatively fair number of combinations and simulate better results, full training can be performed $K$ times as needed. In this work, $K$ is equal to 20 .

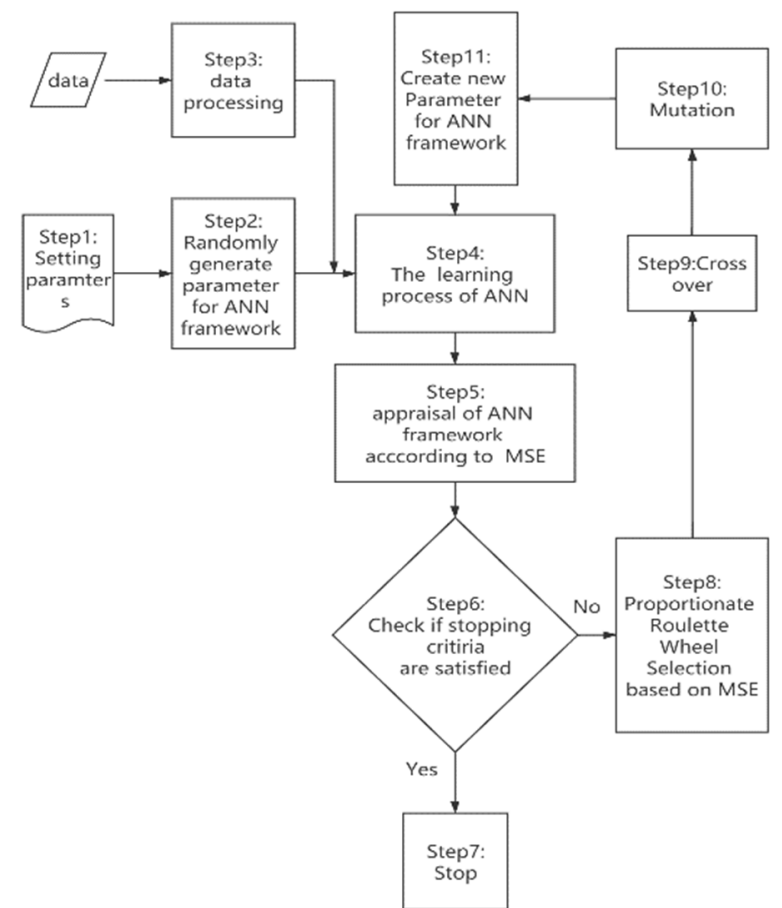

Fig. 1. Flow chart of the GANN.

\subsection{Experimental database for training and validation of the GANN}

Experimental monotonic and cyclic triaxial data is obtained from the database of Karlsruhe sand [9]. Under triaxial monotonic conditions, 25 drained (TMD) and 12 undrained (TMU) tests are available. For triaxial cyclic undrained conditions, 22 tests with stress cycles (TCUI) and 14 tests with strain cycles (TCUE) are available. A part of the data is used as training set and the remaining data as the validation set. The initial void ratio $e_{0}$ and relative density $I_{D 0}$ of the experimental database vary between 0.996 to 0.698 and 0.15 to 0.94 , respectively. The initial confining pressure $p_{0}$ vary between 50 and $400 \mathrm{kPa}$

\subsection{Selection of samples for training of the GANN}

At the beginning of the process, it is difficult to determine which sample combination is the optimum and more representative for training and validation. To explore a relatively fair number of combinations and simulate better results, the method of randomly selecting training samples is adopted. If the number of times the full training $K$ is performed is large enough, e.g., 20 times in this paper, the performance of the ANN is considered acceptable (MSE is less than 0.01 and the regression coefficient $R$ is greater than 0.9 ). The size of the database determines the selection of the $K$ value. After analysing the results of random selection, if necessary, manual selection can be used to further reduce the size of the training set.

\section{Results and analysis of results}

This paper uses the $R$ value to assess the predictive ability of the network. An $R$ value of 1 means a close relationship, 0 a random relationship.

\subsection{Triaxial monotonic drained tests (TMD)}

When the training samples are randomly selected, in the best case of sample combination, then for a number of training samples of $6 / 25$, the performance of the neural result is already acceptable, i.e., $M S E$ error $=0.01$ and $R=0.9696$.

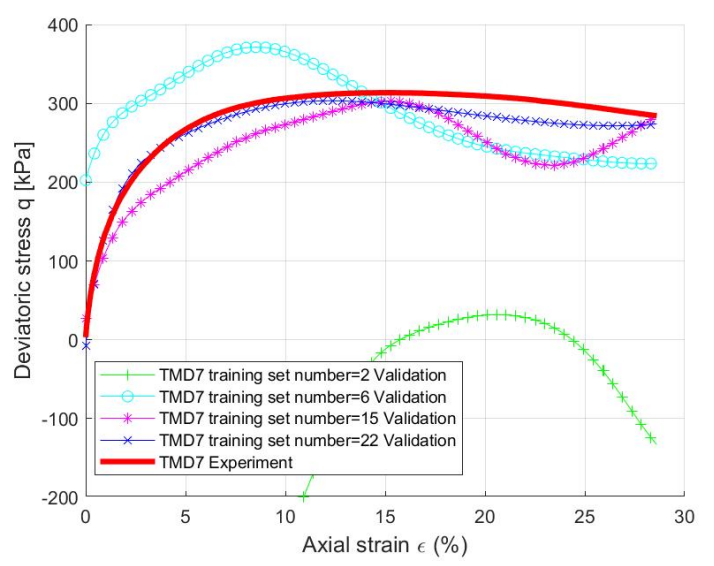

Fig. 2 Deviatoric stress $q$ versus axial strain $\varepsilon 1$ in drained monotonic triaxial tests $\left(I_{D O}=0.51\right.$ and $\left.\mathrm{p}_{0}=100 \mathrm{kPa}\right)$.

The improvement of ANN's prediction capability with the increase of the number of training samples is presented in Fig. 2 and Fig. 3. When the number of 
training samples increases, the validation curve gets closer to the experimental curve as expected. The obtained $R$-values for $M$ equal to 2, 6, 15 and 22 are $0.322,0.7083,0.8944$ and 0.9984 .

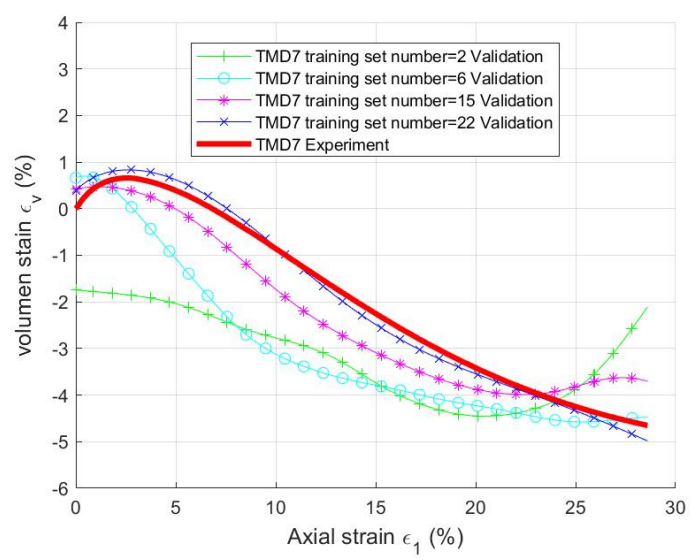

Fig. 3 Volumetric strain $\varepsilon_{v}$ versus axial strain $\varepsilon_{l}$ in drained monotonic triaxial tests $\left(I_{D 0}=0.51\right.$ and $\left.\mathrm{p}_{0}=100 \mathrm{kPa}\right)$.

\subsection{Triaxial monotonic undrained tests (TMU)}

For the TMU tests, in the case of random selection of samples, the number of samples used for training must reach 9/12 to obtain an ANN with good performance $(M S E=0.008$ and $R=0.9606)$. Fig. 4 and Fig. 5 show the performance improvement of the ANN by increasing the number of training samples.

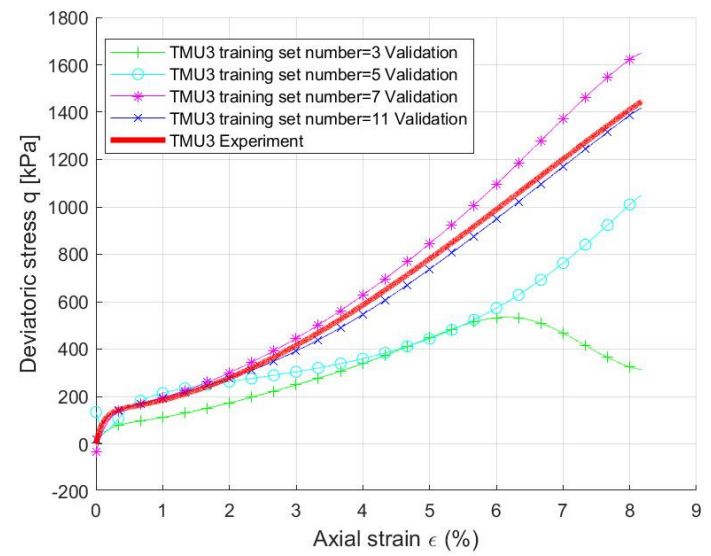

Fig. 4 Deviatoric stress $q$ versus axial strain $\varepsilon_{l}$ in undrained monotonic triaxial tests $\left(I_{D 0}=0.62\right.$ and $\left.\mathrm{p}_{0}=300 \mathrm{kPa}\right)$.

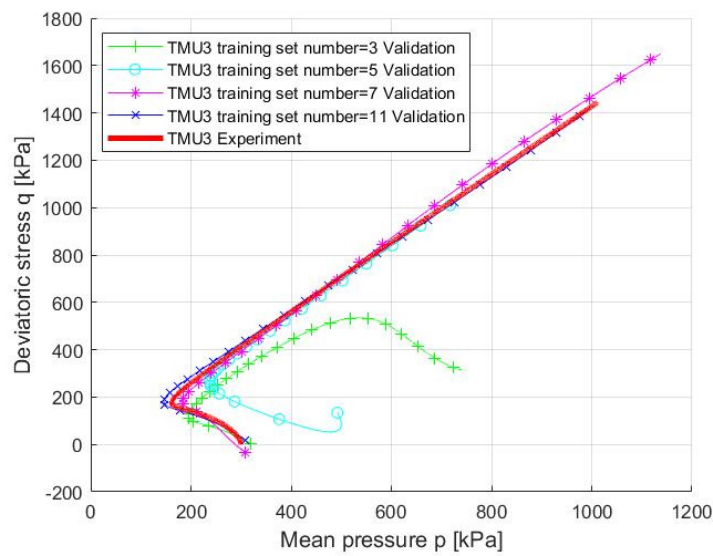

Fig. 5 Deviatoric stress $q$ versus mean pressure $p$ in undrained monotonic triaxial tests $\left(I_{D 0}=0.62\right.$ and $\left.\mathrm{p}_{0}=300 \mathrm{kPa}\right)$.

\subsection{Triaxial cyclic undrained tests with stress cycles (TCUI)}

For the TCUI tests, by random selection, the ANN with $11 / 22$ training set has already good performance, i.e., $M S E$ error $=0.006$ and $R=0.9268$. In order to show the results more clearly, only one validation set is presented in Fig. 6.

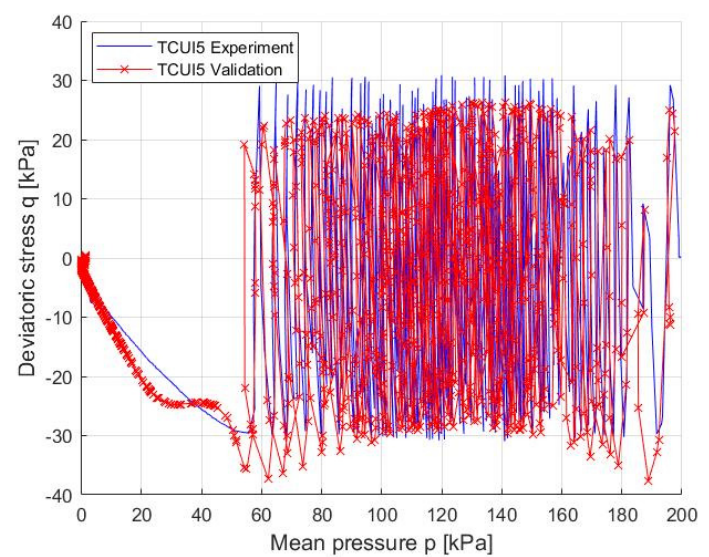

Fig. 6 Effective stress paths in undrained cyclic tests with isotropic initial stresses and stress cycles performed on loose specimens $\left(I_{D 0}=0.26\right.$ and $\left.\mathrm{p}_{0}=200 \mathrm{kPa}\right)$.

We also investigate the cyclic strength ratio $C S R=$ $q^{a m p l} /\left(2 p_{0}\right)$ versus the number of cycles $N$ required to reach the onset of large axial strain generation $\left(\left|\varepsilon_{l}\right|>\right.$ $1 \%$ ), see Fig. 7. As can be seen, the accuracy of the simulation results for dense and loose sand is better than for medium sand. This is related to the selection of the training set, i.e., all loose and part of the dense samples are used as training set and the remaining part of the dense and all medium dense samples are used as validation set. Therefore, before the validation phase, the ANN knows part of the dense data. Nevertheless, the overall simulation results of TCUI are still good and can be improved by including medium dense samples in the training phase.

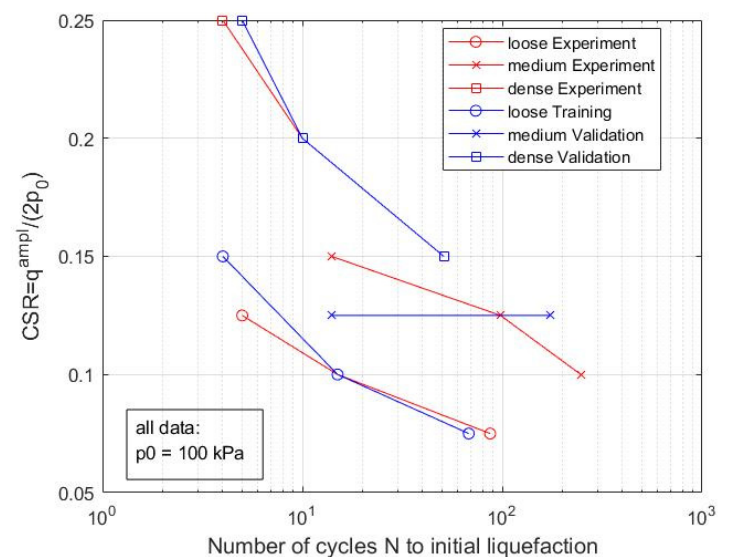

Fig. 7 Amplitude-pressure ratio $q^{a m p l} /\left(2 p_{0}\right)$ vs. the number of cycles $\mathrm{N}$ required to reach the onset of large axial strain generation $(|\varepsilon l|>1 \%),\left(I_{D 0}=0.24-0.87\right.$ and $\left.\mathrm{p}_{0}=100-400 \mathrm{kPa}\right)$. 


\subsection{Triaxial cyclic undrained tests with strain cycles (TCUE)}

In the case of the TCUE tests, $12 / 14$ training set is required to obtain a good performance of the ANN. As can be seen in Fig. 8 and Fig. 9, the ANN can better predict the effective stress paths for relatively small strain cycles. The related $R$ value and MSE are 0.9639 and 0.001 respectively.

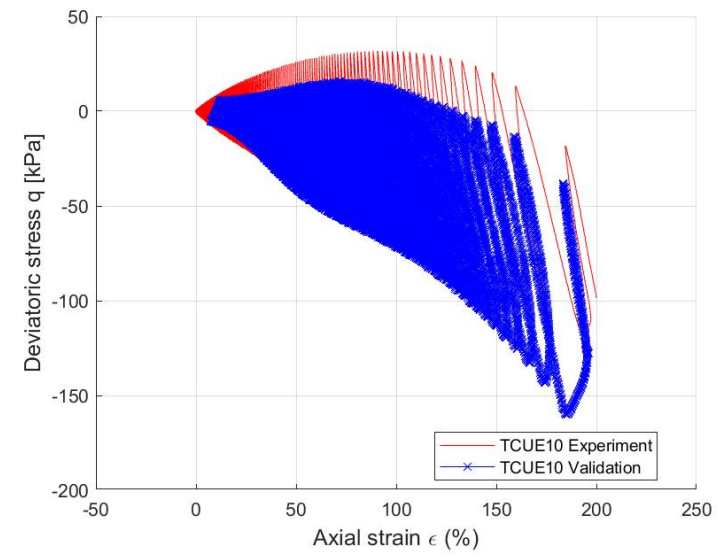

Fig. 8 Effective stress paths in undrained cyclic triaxial tests with relatively small strain cycles $\left(I_{D 0}=0.56\right.$ and $\left.\mathrm{p}_{0}=200 \mathrm{kPa}\right)$.

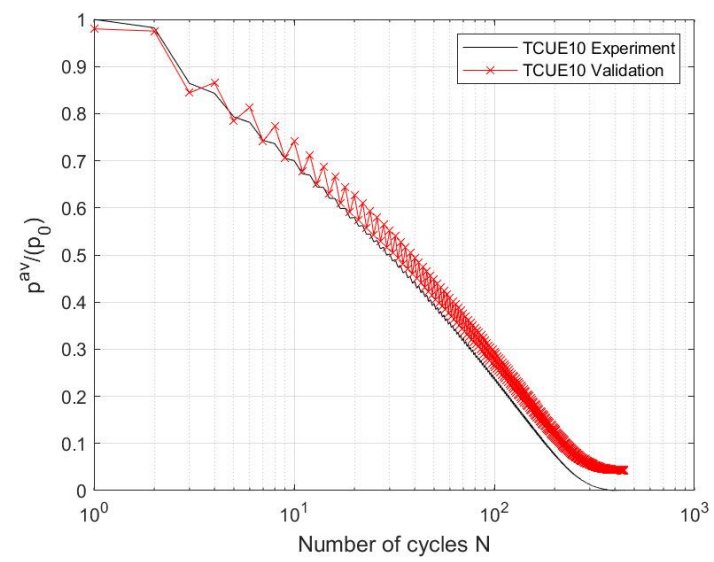

Fig. 9 Relaxation of normalized average mean effective stress $p^{a v}=p_{0}$ with increasing number of cycles in undrained cyclic triaxial tests with prescribed strain amplitude $\left(I_{D 0}=0.56\right.$ and $\mathrm{p}_{0}=200 \mathrm{kPa}$ ).

\section{Conclusions}

We use an ANN algorithm combining BP and GA to simulate the monotonic and cyclic behaviour of Karlsruhe Sand under drained and undrained triaxial conditions. Based on the results we conclude that:

1. When the number of times the full training is performed, $K$ value, is large enough, e.g., 20 in this paper, the performance of the ANN is acceptable (MSE is less than 0.01 and $R$ is greater than 0.9). Manual selection of the training set reflecting the main features of the data can be used to further reduce the number of sets selected for training. Human expert interaction would be required for this selection or a further algorithm could be developed.

2. GANN can effectively simulate the monotonic behaviour of sands under drained and undrained triaxial test. In addition, when TMD and TMU databases are used together for training, GANN can distinguish between the two data sets main features and deliver a good prediction. As TMU tests comprises compression and expansion paths, GANN needs a larger number of TMU training samples to obtain good simulations results as compared with TMD.

3. GANN can also realistically simulate the cyclic behaviour of sands for undrained triaxial test with strain- and stress-controlled cyclic shearing.

The results demonstrate that GANN can realistically simulate the monotonic and cyclic behaviour of sands for different stress paths. Future research comprises the application of ANN instead of constitutive relations (e.g., plasticity, hypoplasticity) to the numerical solution of element tests and boundary value problem in geotechnical Engineering using numerical methods as the Finite Element Method.

\section{References}

[1] K.S. Ti, B.B. Huat, J. Noorzaei, M.S. Jaafar, G.S. Sew, Electron. J. Geotech. Eng. 14, 1-18 (2009)

[2] Bolton, The role of micro-mechanics in soil mechanics (2000).

[3] F.E. Bock, R.C. Aydin, C.J. Cyron, N. Huber, S.R. Kalidindi, B. Klusemann, Front. Mater. 6, 110 (2019)

[4] D. Goldberg, M.S. I., M .Rokonuzzaman, Taylor \& Francis 16, 46-52 (1988)

[5] A.R. Simpson, S.D. Priest, Comput. and Geotech. 15, 1-19 (1993)

[6] H. Ganjidoost, S.J. Mousavi, A. Soroush, Neural Process Lett. 44, 53-79 (2016)

[7] S. Saran, Shallow foundations and soil constitutive laws, CRC Press (2017)

[8] P. Samarajiva, E.J. Macari, W. Wathugala, Int. J. Geomech. 5, 206-217 (2005)

[9] T. Wichtmann, T. Triantafyllidis, Acta Geotech. 11, 739-761 (2016)

[10]X.-S. Yang, A.H. Gandomi, S. Talatahari, A.H. Alavi, Metaheuristics in water, geotechnical and transport engineering, Newnes (2012)

[11] Y. LeCun, Y. Bengio, G. Hinton, Nature 521, 436$444(2015)$ 